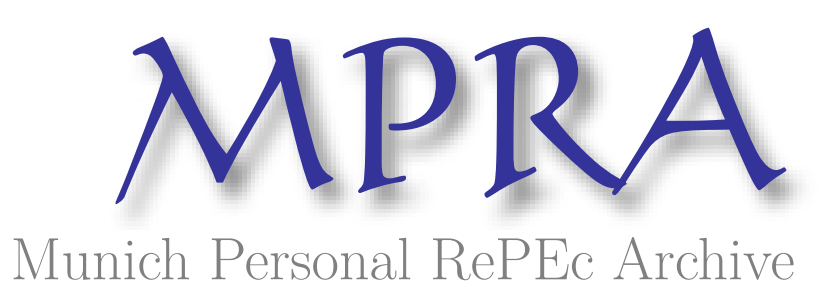

\title{
The Effect of Observability on the Noncontractible Investment of a Regulated Firm
}

Saglam, Ismail

Ankara, Turkey

3 January 2017

Online at https://mpra.ub.uni-muenchen.de/75963/

MPRA Paper No. 75963, posted 04 Jan 2017 08:44 UTC 


\title{
The Effect of Observability on the Noncontractible Investment of a Regulated Firm
}

\author{
Ismail Saglam
}

\begin{abstract}
We study the effect of observability on the noncontractible investment of a regulated firm with private marginal cost information. We show that the observability reduces investment, pointing to the regulated firm's prevention of ratcheting. This result, which is in line with an earlier finding of Tirole (1986) obtained in a bargaining model of procurement with two-sided asymmetric information, reveals that 'underinvestment due to observability' is independent of whether only the investing firm or all of the parties affected by its investment decision have some private information.
\end{abstract}

Keywords: Monopoly; Regulation; Investment; Observability; Asymmetric Information JEL Classification Numbers: D82, L51, O32 


\section{Introduction}

A pioneering work of Tirole (1986) showed that the noncontractible investment of a firm is lower when it is observable than when it is not. This finding was obtained in a two-period procurement model where a single project is produced and sold by a single firm to a single sponsor. The firm, in the first period, invests in reducing the costs of the project. At the beginning of the second period, the sponsor learns its private value for the project while the firm privately learns the costs it will face if it realizes the project. Then takes place a noncooperative bargaining where the firm and the sponsor determine whether to trade and the price contingent on trade. According to Tirole (1986), why observability leads to underinvestment in this model can be explained by the so called 'information effect', which only arises when investment is observable since in that case the firm can not only influence its cost distribution but also the sponsor's beliefs about it. As the sponsor's price under observability decreases with the sponsor's beliefs about the firm's investment, more optimistic beliefs make the sponsor less agreeable in the bargaining process in the second period, leading the firm to reduce its investment below the level it would choose under unobservability.

One may here ask whether or not Tirole's (1986) result of underinvestment due to observability is sensitive to his modelling assumption that both the firm and the sponsor have some private information about the project and can thus make a noncooperative bargaining for trade. To answer this question, we will study in this paper whether observability affects the noncontractible investment decision of a regulated firm in a principal-agent model where 'only' the agent, i.e. the investing firm, holds some private information about the regulated environment.

While our research question is novel to the best of our knowledge, investment of a firm in a principal-agent framework has been extensively studied in the regulation literature, where the closest works to our study are Baron and Besanko (1984) and Laffont and Tirole (1993). Both of these works consider the case of unobservable and noncontractible investment as well as the case of observable and contractible invest-

ment. However, neither of them studies the case of observable and noncontractible investment, which as mentioned by Laffont and Tirole (1993, p. 88) may arise as a 
third possibility when "The regulator might observe investment but not be able to provide evidence that is accurate enough for a court in charge of enforcing the regulatory contract." Our paper will attempt to consider this unexplored case together with the earlier studied case of unobservable and noncontractible investment so as to identify the effect of observability on noncontractible investment in a principal-agent model with 'one-sided' informational asymmetry.

For tractability of our results, we will consider a simpler model than the models considered by Baron and Besanko (1984) and Laffont and Tirole (1993). Basically, we will integrate investment with Baron and Myerson's (B-M) (1982) well-known model that optimally regulates -through an incentive-compatible policy- a monopolist with unknown production costs. ${ }^{1}$ In more detail, we will consider prior to the production stage in the B-M model an investment stage in which the monopolist has access to an investment (or research and development) technology determined by a fixed parameter $\gamma \in(0,1)$ and a choice variable $\rho \in[0,1)$. This technology will reduce the monopolist's private marginal cost, say $\theta$, to $\gamma \theta$ with probability $\rho$. The parameter $\gamma$ will be called the improvement due to (successful) investment. On the other hand, the variable $\rho$ will be determined by the level of investment, and will be called the probability of success or the level of investment interchangeably. We will close the model by defining a cost function for investment.

For the model we have described above, we will assume that the regulator can not contract on investment, implying that the value of the parameter $\rho$ will be freely determined by the monopolist. However, as in the B-M model without investment, the regulator will be able to contract on the price and output of the product. Regarding the regulator's knowledge about investment, we will consider two possibilities: The first one is that the regulator is ex-ante aware of the investment technology accessed by the monopolist and is ex-post able to observe investment (say through mandated reporting and auditing); i.e., she learns about the investment technology $(\gamma, \rho)$ and the actual value of the parameter $\gamma$ before investment takes place, and observes the

\footnotetext{
${ }^{1}$ Thus, unlike Baron and Besanko (1984) we will consider a single-period model, and unlike Laffont and Tirole (1993) we will assume away any (managerial) efforts for cost reduction after the investment stage.
} 
actual value of the parameter $\rho$ after investment takes place. ${ }^{2}$ The second possibility is that the regulator is never aware of the investment technology $(\gamma, \rho)$ and she never observes $\gamma$ or $\rho$. We will study these two possibilities respectively in Section 3.1 and Section 3.2, after presenting our model in Section 2. In Section 3.3 we will compare the optimal investment decisions calculated in Sections 3.1 and 3.2, yielding our main result that a regulated monopolist invests less (more) when investment is observable (unobservable). Furthermore, in Section 3.4 we will show that in situations where the social welfare attaches a sufficiently high weight to the monopolist welfare, the unobservable investment of a regulated monopolist is even higher than the investment of an unregulated monopolist. Finally, we will conclude in Section 4.

\section{Model}

We will first present our model without investment, which is a slightly simplified version of the regulation model of B-M. Consider a monopolist facing the cost function

$$
C(q, \theta)=K+\theta q \quad \text { if } q>0, \quad \text { and } \quad C(0, \theta)=0,
$$

where $K \geq 0$ denotes the fixed cost of producing any positive quantity of output and $\theta$ denotes the privately known marginal cost lying in the interval $\left(0, \theta_{1}\right]$, with $\theta_{1}>0$. The demand faced by the monopolist at the price $p$ is denoted by $D(p)$ and satisfies

$$
D(p)=D_{0}-D_{1} p, \quad \text { for all } p \in\left[0, D_{0} / D_{1}\right],
$$

\footnotetext{
${ }^{2}$ The assumption that the regulator is ex-ante informed about the improvement level of investment, or relatedly the parameter $\gamma$, will simplify our research problem quite a lot. In situations this assumption does not hold, the regulated firm would “..., recognize that any investment it may make to increase its efficiency will result in the regulator seeking information about its post-investment cost structure in order to establish prices appropriate for the new level of efficiency. The manner in which the regulator is expected to use the information to be obtained in the future thus affects the firm's incentive to make efficiency-enhancing investments and hence creates a moral hazard problem." [Baron and Besanko (1984, p. 268).]
} 
where $D_{0}, D_{1}>0$ and $D\left(\theta_{1}\right)>0 .{ }^{3}$ We restrict ourselves to this simple form of demand to analyze, in Section 3, the effect of demand shocks (or changes in the maximal size of demand, $D_{0}$ ) on the optimal level of investment. Formally, we say that there is a demand shock to the monopolist (possibly caused by a change in consumers' income or taste) if $D_{0}$ changes.

Given the demand function $D$, the total value to consumers of an output of quantity $q$ is

$$
V(q)=\int_{0}^{q} D^{-1}(x) d x,
$$

and the consumer surplus is $V(q)-D^{-1}(q) q$.

The price and quantity in the monopolistic market will be determined by a regulatory authority. While the regulator does not know the actual value of the marginal cost of the monopolist, she has prior beliefs about it, represented by the density function $f$, which is positive and continuous over its support $\left(0, \theta_{1}\right]$. Correspondingly, $F$ denotes the cumulative distribution function. It is assumed that the only informational asymmetry in the above model is about $\theta$; everything else is symmetrically known.

\subsection{Regulatory Policy of B-M}

The class of regulatory policies considered by B-M for the above-described monopolistic market (with no investment possibility) involves outcome functions $\langle r, p, q, s\rangle$ that will be characterized below. Announcing these four functions, the regulator asks the monopolist to report its marginal cost parameter. If the monopolist reports $\tilde{\theta}$ as its marginal cost, $r(\tilde{\theta})$ is the probability that it is allowed to sell, $p(\tilde{\theta})$ and $q(\tilde{\theta})$ are the regulated price and quantity of the product respectively, and $s(\tilde{\theta})$ is the expected value of the subsidy the monopolist will receive conditional on the probability that it is allowed to sell. Then, the expected profit of the monopolist, when it reports $\tilde{\theta}$

\footnotetext{
${ }^{3}$ We also assume for convenience that the parameters $D_{0}$ and $D_{1}$ are such that demand is always nonnegative at all regulated prices in Section 2 and Section 3.
} 
as its marginal cost while it is actually $\theta$, can be written as

$$
\pi(\tilde{\theta}, \theta)=[p(\tilde{\theta}) q(\tilde{\theta})-C(q(\tilde{\theta}), \theta)] r(\tilde{\theta})+s(\tilde{\theta}) .
$$

A regulatory policy $\langle r, p, q, s\rangle$ is called feasible if it satisfies the following conditions for all $\theta \in\left(0, \theta_{1}\right]$ :

(i) $r(\theta)$ is a probability function, i.e.,

$$
0 \leq r(\theta) \leq 1,
$$

(ii) $p(\theta)$ and $q(\theta)$ are consistent with each other on the demand curve, i.e.,

$$
q(\theta)=D(p(\theta)),
$$

(iii) the regulatory policy is incentive-compatible (truthful revelation is optimal) for the monopolist, i.e.,

$$
\pi(\theta, \theta) \geq \pi(\tilde{\theta}, \theta), \text { for all } \tilde{\theta} \in\left(0, \theta_{1}\right],
$$

(iv) the regulatory policy is individually rational for the monopolist under truthful revelation, i.e.,

$$
\pi(\theta, \theta) \geq 0 .
$$

Now, consider any $\theta \in\left(0, \theta_{1}\right]$. Given a feasible regulatory policy $\langle r, p, q, s\rangle$, the consumer welfare (consumer surplus net of the subsidy paid to the monopolist) and the producer welfare (operational profit plus subsidy paid by consumers) become

$$
C W(\theta)=[V(q(\theta))-p(\theta) q(\theta)] r(\theta)-s(\theta),
$$

and

$$
\pi(\theta) \equiv \pi(\theta, \theta)=[p(\theta) q(\theta)-C(q(\theta), \theta)] r(\theta)+s(\theta),
$$

respectively. The social welfare $S W(\theta)$ is defined to be a weighted average of consumer welfare $C W(\theta)$ and the producer welfare $\pi(\theta)$. Formally,

$$
\begin{aligned}
S W(\theta) & =C W(\theta)+\alpha \pi(\theta) \\
& =[V(q(\theta))-C(q(\theta), \theta))] r(\theta)-(1-\alpha) \pi(\theta),
\end{aligned}
$$


where $\alpha \in[0,1]$ is the weight parameter.

The problem of the regulator, who is uninformed about $\theta$, is to choose a feasible regulatory policy that will lead to the highest expected value of $S W(\theta)$ in (11), conditional on her prior beliefs about $\theta$. Formally, the regulator's objective is to find optimal policy functions that will solve

$$
\max _{r(.), p(.), q(.), s(.)} \int_{0}^{\theta_{1}} S W(\theta) f(\theta) d \theta \text { subject to }(5)-(8) .
$$

Before stating the solution to the above problem, we will make the following restriction on the regulator's beliefs for the tractability of our analysis in Section 3.4

Assumption 1. $F(\theta) / f(\theta)$ is nondecreasing in $\theta \in\left(0, \theta_{1}\right]$.

Proposition 1. (Baron and Myerson, 1982) Let Assumption 1 hold. Then, the solution to the regulator's problem in (12) is given by the optimal policy $\langle\bar{r}, \bar{p}$, $\bar{q}, \bar{s}\rangle$ satisfying equations (13)-(16) for all $\theta \in\left(0, \theta_{1}\right]$ :

$$
\begin{aligned}
& \bar{p}(\theta)=\theta+(1-\alpha) \frac{F(\theta)}{f(\theta)} \\
& \bar{q}(\theta)=D(\bar{p}(\theta)) \\
& \bar{r}(\theta)= \begin{cases}1 & \text { if } V(\bar{q}(\theta))-\bar{p}(\theta) \bar{q}(\theta) \geq K \\
0 & \text { otherwise }\end{cases} \\
& \bar{s}(\theta)=[K+\theta \bar{q}(\theta)-\bar{p}(\theta) \bar{q}(\theta)] \bar{r}(\theta)+\int_{\theta}^{\theta_{1}} \bar{r}(x) \bar{q}(x) d x
\end{aligned}
$$

Note in the above proposition that inserting the optimal subsidy (16) into the profit equation (10) yields

$$
\pi(\theta)=\int_{\theta}^{\theta_{1}} \bar{r}(x) \bar{q}(x) d x
$$

\footnotetext{
${ }^{4}$ The optimal regulatory policy in Baron and Myerson (1982) is characterized without using Assumption 1.
} 
implying that the profit of the monopolist is purely informational rent. It is clear from (17) that this rent will be higher, the lower is the monopolist's marginal cost of production, $\theta$. So, there is room for the monopolist in the B-M model to make cost-reducing investment.

\subsection{Integrating the B-M Model with Investment}

Consider a pre-regulatory stage (called investment stage) in which the monopolist has access to an investment (or simply research and development) technology to reduce its production costs. This technology is described by a variable $\rho \in[0,1)$ and a parameter $\gamma \in(0,1)$. Basically, this technology reduces a given marginal cost of production $\theta$ to the level $\gamma \theta$ with probability $\rho$. (We exclude $\rho=1$, as sure improvement will be assumed to be infinitely costly.) Since one may expect higher likelihoods of improvement with a higher level of investment, the variable $\rho$ will be called the level of investment, for brevity. On the other hand, $\gamma$ will be called the improvement parameter, since the lower $\gamma$ is, the higher the (production) cost reduction obtained from a successful investment. We assume that $\gamma$ is a fixed parameter, while $\rho$ is a control variable for the firm. We will leave stating the nature of the regulator's knowledge about the parameters $\gamma$ and $\rho$ to Section 3 .

We will close our model by introducing $R(\rho, \gamma)$ to denote the cost of using the investment technology $(\rho, \gamma)$. We assume that the function $R$ is twice continuously differentiable with respect to both of its arguments and satisfies the following.

Assumption 2. $R(0, \gamma)=0$ (there are no sunk costs of investment).

Assumption 3. $R_{\rho}(\rho, \gamma)>0$ for all $\rho \in(0,1)$ (Investment cost is increasing in the level of activities).

Assumption 4. $R_{\rho}(0, \gamma)=0$ (marginal cost is zero at zero investment).

Assumption 5. $\lim _{\rho \uparrow 1} R_{\rho}(\rho, \gamma)=\infty$ (improvement with certainty increases costs unboundedly). 
Assumption 6. $R_{\rho \rho}(\rho, \gamma)>0$ for all $\rho \in[0,1)$ (investment cost is strictly convex in the level of activities).

Apart from $\rho$ and $\gamma$, no parameter in our model affects the cost function $R(.,$.$) .$ While this function is known to the monopolist, it is completely unknown to the regulator. ${ }^{5}$ Because of this informational asymmetry, the regulator will not be able to optimally revise its optimal contract in (13)-(16) to influence investment of the monopolist, even when she is aware of the possibility of investment.

\section{Results}

We will assume throughout the rest of this paper that the regulator can not contract on investment. So, the level of investment, i.e., the value of the parameter $\rho$, will be determined by the regulated monopolist. One can here suppose that we consider an environment where the legal system does not allow the regulatory authority to directly control investment or that the regulator cannot verify investment for a third party (court) even when it is observable. In this environment, we will consider two possibilities in the following two subsections, namely the case where investment is observable to the regulator and the case where it is unobservable. (We will relegate the proofs of all results to Appendix.)

\subsection{Observable Investment}

Here, we will consider an environment where the regulator is ex-ante aware of the monopolist's investment technology $(\gamma, \rho)$ and knows the actual value of $\gamma$, the effi-

\footnotetext{
${ }^{5}$ The asymmetric assumption that the regulator is completely uninformed about investment costs while she has incomplete information about production costs should make sense, once we observe that unlike investment costs, production costs can be partially or completely inferred or verified by the regulator through inspecting the quality of the product. See Sappington (1982) for an investment model where the regulator (as well as the firm) has imperfect information about the technology of cost reduction.
} 
ciency of investment. The regulator can also ex-post observe the monopolist's choice of $\rho$, the level of investment. Given her awareness about the investment technology, the regulator will condition -before she observes investment of the monopolist- her beliefs about the marginal cost of production on the parameter $\rho$; i.e. she will update her prior beliefs $f($.$) to f(. \mid \rho)$ for $\rho \in[0,1)$. After the regulator observes the realized investment of the monopolist, say the value $\rho^{*}$, her conditional beliefs will accordingly change to $f\left(. \mid \rho^{*}\right)$. Given these observations, we will now describe the whole regulatory process in five stages:

Stage 1: The regulator learns that the monopolist has access to an investment technology described by the pair of parameters $\rho \in[0,1)$ and $\gamma \in(0,1)$ and that this technology will reduce the marginal cost of the monopolist by $(1-\gamma) 100 \%$ with probability $\rho$. (At this stage, the regulator knows the actual value of $\gamma$; but she does not know the actual value of $\rho$.)

Stage 2: The regulator announces that the regulatory policy will be given by the B-M regulatory policy modified with respect to the conditional beliefs $f(. \mid \rho)$, where the actual value of $\rho$ will be observed by the regulator in stage 4. (We will state this modified regulatory policy in Proposition 2 by equations (22)-(25).)

Stage 3: In response to the announced regulatory policy, the monopolist with the private marginal cost $\theta$ determines and realizes the level of investment as $\rho^{*}(\theta)$ and learns whether its post-investment marginal cost is $\theta$ or $\gamma \theta$.

Stage 4: The regulator observes $\rho^{*}(\theta)$ and announces $f\left(. \mid \rho^{*}(\theta)\right)$ as her actual beliefs.

Stage 5: The monopolist reports its post-investment marginal cost ( $\gamma \theta$ if the investment was successful, and $\theta$ otherwise), and the corresponding regulatory outcome is calculated and implemented by the regulator.

With the regulator moving first in the investment game (stage 2) of the above process, we implicitly assume that the regulator commits to particular policies that could influence subsequent behavior of the monopolist (stage 3). If instead the regulator could revise its choice after the monopolist chooses its investment, different outcomes might occur. We implicitly consider a regulatory environment that involves 
adjustment costs, such as the difficulty of getting political approval, preventing such a policy redesign.

Now we are ready to characterize the outcome of the above process. We will start deriving the optimal policy the regulator will announce in the second stage. As the regulator has become, in the first stage, aware of the investment technology that will used by the monopolist, she can update her prior beliefs $f(\theta)$ at each $\theta \in\left(0, \theta_{1}\right)$ to the posterior beliefs $f(\theta \mid \rho)$ for each possible value of $\rho \in[0,1)$ as follows:

$$
f(\theta \mid \rho)= \begin{cases}\frac{\rho}{\gamma} f(\theta / \gamma)+(1-\rho) f(\theta) & \text { if } 0<\theta \leq \gamma \theta_{1} \\ (1-\rho) f(\theta) & \text { if } \gamma \theta_{1}<\theta \leq \theta_{1}\end{cases}
$$

Corresponding to the density function $f(\theta \mid \rho)$, the cumulative distribution and the inverse hazard rate functions can be calculated as

$$
F(\theta \mid \rho)= \begin{cases}\rho F(\theta / \gamma)+(1-\rho) F(\theta) & \text { if } \quad 0<\theta \leq \gamma \theta_{1}, \\ \rho+(1-\rho) F(\theta) & \text { if } \gamma \theta_{1}<\theta \leq \theta_{1},\end{cases}
$$

and

$$
\frac{F(\theta \mid \rho)}{f(\theta \mid \rho)}= \begin{cases}\frac{\rho F(\theta / \gamma)+(1-\rho) F(\theta)}{\frac{\rho}{\gamma} f(\theta / \gamma)+(1-\rho) f(\theta)} & \text { if } \quad 0<\theta \leq \gamma \theta_{1}, \\ \frac{\rho}{(1-\rho) f(\theta)}+\frac{F(\theta)}{f(\theta)} & \text { if } \gamma \theta_{1}<\theta \leq \theta_{1},\end{cases}
$$

respectively. When $\rho$ is zero (the case of no investment), we have $f(\theta \mid \rho)=f(\theta)$, $F(\theta \mid \rho)=F(\theta)$, and $F(\theta \mid \rho) / f(\theta \mid \rho)=F(\theta) / f(\theta)$, as expected.

The regulator's objective is to find optimal policy functions that will maximize the expected social welfare under the updated beliefs $f(\theta \mid \rho)$ :

$$
\max _{r(.), p(.), q(.), s(.)} \int_{0}^{\theta_{1}} S W(\theta) f(\theta \mid \rho) d \theta \text { subject to }(5)-(8) .
$$

One can immediately wonder whether the regulatory policy given by (13)-(16) would solve the problem in (21) whenever the inverse hazard rate $F(\theta) / f(\theta)$ in that policy was replaced by $F(\theta \mid \rho) / f(\theta \mid \rho)$. The answer is 'yes' if $F(\theta \mid \rho) / f(\theta \mid \rho)$ 
is nondecreasing in $\theta \cdot{ }^{6}$ For this property to always hold, Assumption 1 will be strengthened as follows.

Assumption 7. The density $f(\theta)$ is nonincreasing in $\theta \in\left(0, \theta_{1}\right]$.

Also, the following lemma will be instrumental to solve (21).

Lemma 1. Let Assumption 7 hold. Then, for all $\rho \in[0,1)$, the rate $F(\theta \mid \rho) / f(\theta \mid \rho)$ is increasing in $\theta \in\left(0, \theta_{1}\right]$.

We can now state the optimal regulatory policy under observable investment.

Proposition 2. Let Assumption 7 hold. Then, for any $\rho \in[0,1)$, the solution to the regulator's problem in (21) is given by the optimal policy $\left\langle\bar{r}^{\rho}, \bar{p}^{\rho}, \bar{q}^{\rho}, \bar{s}^{\rho}\right\rangle$ satisfying equations (22)-(25) for all $\theta \in\left(0, \theta_{1}\right]$ :

$$
\begin{aligned}
& \bar{p}^{\rho}(\theta)=\theta+(1-\alpha) \frac{F(\theta \mid \rho)}{f(\theta \mid \rho)} \\
& \bar{q}^{\rho}(\theta)=D\left(\bar{p}^{\rho}(\theta)\right) \\
& \bar{r}^{\rho}(\theta)= \begin{cases}1 & \text { if } V\left(\bar{q}^{\rho}(\theta)\right)-\bar{p}^{\rho}(\theta) \bar{q}^{\rho}(\theta) \geq K \\
0 & \text { otherwise }\end{cases} \\
& \bar{s}^{\rho}(\theta)=\left[K+\theta \bar{q}^{\rho}(\theta)-\bar{p}^{\rho}(\theta) \bar{q}^{\rho}(\theta)\right] \bar{r}^{\rho}(\theta)+\int_{\theta}^{\theta_{1}} \bar{r}^{\rho}(x) \bar{q}^{\rho}(x) d x
\end{aligned}
$$

Apparently, when $\rho=0$, the optimal policy in the above proposition reduces to the optimal policy (13)-(16) proposed by B-M, i.e., $\left\langle\bar{p}^{0}, \bar{q}^{0}, \bar{r}^{0}, \bar{s}^{0}\right\rangle=\langle\bar{p}, \bar{q}, \bar{r}, \bar{s}\rangle$. In fact, we could have derived Proposition 1 as a direct corollary to Proposition 2. To study the effect of $\rho$ on the regulatory outcome, the following assumption will be

\footnotetext{
${ }^{6}$ Mimicking the proof of Proposition 1, which was provided by B-M for the case of $\rho=0$ in the extended model of ours, one can easily show that the incentive-compatibility condition in (7) is satisfied if the inverse hazard rate $F(\theta \mid \rho) / f(\theta \mid \rho)$ is nondecreasing.
} 
useful.

Assumption 8. $F(\gamma \theta) / f(\gamma \theta)<\gamma F(\theta) / f(\theta)$, for all $\theta \in\left(0, \theta_{1}\right]$.

Assumption 8 requires that the gross rate of change in the inverse hazard rate due to investment is bounded from above by the parameter $\gamma$. One can easily check that this assumption is satisfied if $F(\theta) / f(\theta)$ is strictly convex over $\theta \in[0, \infty)$. Assumptions 7 and 8 will be instrumental for the below lemma as well as for a corollary to Proposition 2.

Lemma 2. Let Assumptions 7 and 8 hold. Then, for all $\theta \in\left(0, \theta_{1}\right]$, the inverse hazard rate $F(\theta \mid \rho) / f(\theta \mid \rho)$ is (i) increasing in $\rho \in[0,1)$; (ii) convex in $\rho \in[0,1)$.

Corollary 1. Let Assumptions 7 and 8 hold. Then, for all $\theta \in\left(0, \theta_{1}\right]$ and all $\alpha \in[0,1)$, the regulated output, $\bar{q}^{\rho}(\theta)$, is decreasing in $\rho \in[0,1)$.

The above result follows from the fact that with a higher level of investment, the inverse hazard rate, i.e., the marginal informational cost, also becomes higher, as ensured by Lemma 2(i). So, in situations where the regulated price depends on the marginal informational cost (i.e., the cases where $\alpha \neq 1$ ), the regulated price will be higher, while the regulated output will be lower, with an increase in investment. Admittedly, this result may seem quite counterintuitive at first sight: If we expect that the investment of the monopolist will reduce its marginal cost with some known probability, should not we then also expect its output to rise, after all? The answer would obviously be 'yes' in the symmetric information case where the whole social surplus goes to consumers and resultingly the socially optimal output, under regulation, becomes consistent with marginal cost pricing. In the asymmetric information case, however, the social surplus is shared between consumers and the regulated monopolist, as the latter always earns a positive informational rent unless $\alpha=1$. If there was no change in the output schedule in response to investment, the expected informational rent of the regulated monopolist would increase as it is 
likely that its reported marginal cost would become lower thanks to investment. The regulator can suppress the said increase in the informational rent only by reducing the monopolist's output schedule (i.e., the marginal informational rent) as suggested by Corollary 1.

We will simplify the rest of our analysis, by the following assumption. (As we will need this assumption in Section 3.2 and Section 3.4 for the case of $\rho=0$ only, we will state it here for each $\rho$ separately.)

Assumption 9- $[\rho] . \quad V\left(\bar{q}^{\rho}\left(\theta_{1}\right)\right)-\bar{p}^{\rho}\left(\theta_{1}\right) \bar{q}^{\rho}\left(\theta_{1}\right)>K$.

To see the consequence of making the above assumption, we should note that for all $\theta \in\left(0, \theta_{1}\right]$

$$
\frac{d\left[V\left(\bar{q}^{\rho}(\theta)\right)-\bar{p}^{\rho}(\theta) \bar{q}^{\rho}(\theta)\right]}{d \theta}=-\frac{d \bar{p}^{\rho}(\theta)}{d \theta} \bar{q}^{\rho}(\theta)<0,
$$

implying that consumer surplus is decreasing in $\theta \in\left(0, \theta_{1}\right]$. Thus, Assumption 9-[ $\left.\rho\right]$ along with equation (24) will guarantee that when the level of investment is equal to $\rho$, the monopolist will always be allowed to produce, i.e., $\bar{r}^{\rho}()=$.1 .

After the regulator has announced the regulatory policy (22)-(25) in Stage 2, the monopolist will choose, in Stage 3, the level of investment. Let $\pi(\theta, \rho)$ denote the profit of the monopolist if its marginal cost is $\theta$ and the realized investment is $\rho$. Thus,

$$
\pi(\theta, \rho)=\left[\bar{p}^{\rho}(\theta) \bar{q}^{\rho}(\theta)-C\left(\bar{q}^{\rho}(\theta), \theta\right)\right] \bar{r}^{\rho}(\theta)+\bar{s}^{\rho}(\theta)-R(\rho, \gamma)
$$

When Assumption 9-[$]$ holds, inserting (22)-(25) into (27) yields

$$
\pi(\theta, \rho)=\int_{\theta}^{\theta_{1}} \bar{q}^{\rho}(x) d x-R(\rho, \gamma) .
$$

Likewise, $\pi(\gamma \theta, \rho)$ will denote the profit of the monopolist if its marginal cost is $\gamma \theta$ and the realized investment is $\rho$. Then, the expected profit $\pi^{e}(\theta, \rho)$ of the monopolist when its marginal $\operatorname{cost} \theta$ is reduced to $\gamma \theta$ with probability $\rho$ can be written as

$$
\pi^{e}(\theta, \rho)=\rho \pi(\gamma \theta, \rho)+(1-\rho) \pi(\theta, \rho)
$$


or simply

$$
\pi^{e}(\theta, \rho)=B(\theta, \rho)-R(\rho, \gamma)
$$

with

$$
B(\theta, \rho)=\int_{\theta}^{\theta_{1}} \bar{q}^{\rho}(x) d x+\rho \int_{\gamma \theta}^{\theta} \bar{q}^{\rho}(x) d x
$$

denoting the expected benefit of the monopolist. The first term in equation (31) is the (sure) informational rent obtained by the monopolist irrespective of the success of investment, whereas the second term is its (expected) additional informational rent obtained when the marginal cost is reduced from $\theta$ to $\gamma \theta$ with probability $\rho$.

The monopolist will decide to make investment $(\rho>0)$ only if the resulting expected profit exceeds profit under no investment $(\rho=0)$, i.e.,

$$
\pi^{e}(\theta, \rho)-\pi^{e}(\theta, 0)=\int_{\theta}^{\theta_{1}}\left[\bar{q}^{\rho}(x)-\bar{q}^{0}(x)\right] d x+\rho \int_{\gamma \theta}^{\theta} \bar{q}^{\rho}(x) d x-R(\rho, \gamma) \geq 0,
$$

where we have used $R(0, \gamma)=0$ by Assumption 2. Under the above condition, the monopolist's problem of investment can be written as follows:

$$
\max _{\rho \in[0,1)} \pi^{e}(\theta, \rho) \quad \text { subject to }(32)
$$

Let $\rho^{*}(\theta)$ denote the solution to the above problem. When $\rho^{*}(\theta)$ is an interior solution, it satisfies the first-order condition

$$
B_{\rho}\left(\theta, \rho^{*}(\theta)\right)=R_{\rho}\left(\rho^{*}(\theta), \gamma\right)
$$

where

$$
B_{\rho}(\theta, \rho)=\int_{\theta}^{\theta_{1}} \bar{q}_{\rho}^{\rho}(x) d x+\int_{\gamma \theta}^{\theta} \bar{q}^{\rho}(x) d x+\rho \int_{\gamma \theta}^{\theta} \bar{q}_{\rho}^{\rho}(x) d x
$$

for any $\rho \in[0,1)$. In the above equation, the second integral is always positive, whereas the first and third integrals are negative unless $\alpha=1$ (by Corollary 1). Therefore, the sign of $B_{\rho}(\theta, \rho)$ is, in general, ambiguous. For arbitrarily small values 
of $\rho$, we will get rid of this ambiguity by assuming the following.

Assumption 10. $B_{\rho}(\theta, 0)>0$ for all $\theta \in\left(0, \theta_{1}\right]$.

Note that given equation (35), Assumption 10 requires

$$
\left.\left(\int_{\theta}^{\theta_{1}} \bar{q}_{\rho}^{\rho}(x) d x+\int_{\gamma \theta}^{\theta} \bar{q}^{\rho}(x) d x\right)\right|_{\rho=0}>0
$$

for all $\theta \in\left(0, \theta_{1}\right]$. Below, we show that this condition is satisfied if the social welfare attaches a sufficiently high weight to the monopolist welfare.

Remark 1. Let Assumption 9-[0] hold. Then, Assumption 10 will be satisfied if $\alpha$ is sufficiently close to 1.

The following Lemma will be instrumental for the rest of our results in Section 3.1.

Lemma 3. Pick any $\rho \in[0,1)$. Let Assumptions 6-8 and Assumption 9-[ $\rho]$ hold. Then, $\pi_{\rho \rho}^{e}(\theta, \rho)<0$ for all $\theta \in\left(0, \theta_{1}\right]$.

Now, we can state our first characterization result.

Proposition 3. Let Assumptions 2-8 and 10 hold and also Assumption 9- $[\rho]$ hold for all $\rho \in[0,1)$. Then, for all $\theta \in\left(0, \theta_{1}\right]$, the optimal level of observable investment, $\rho^{*}(\theta)$, for the monopolist is unique, lies in $(0,1)$, and satisfies

$$
\left.\left(\int_{\theta}^{\theta_{1}} \bar{q}_{\rho}^{\rho}(x) d x+\int_{\gamma \theta}^{\theta} \bar{q}^{\rho}(x) d x+\rho \int_{\gamma \theta}^{\theta} \bar{q}_{\rho}^{\rho}(x) d x\right)\right|_{\rho=\rho^{*}(\theta)}=R_{\rho}\left(\rho^{*}(\theta), \gamma\right) .
$$

Figure 1 illustrates how to graphically obtain the optimal level of observable investment, $\rho^{*}(\theta)$, for the monopolist. Note that if $\alpha \neq 1$, the marginal benefit curve $B_{\rho}(\theta, \rho)$ is given by the downward sloping curve in this figure. For this case, we find $\rho^{*}(\theta)$ at the intersection of the marginal benefit and cost (green and red) curves. On the other hand, if $\alpha=1$, the regulated output function becomes independent 
of $\rho$, since $\bar{q}^{\rho}()=.\bar{q}()=.D($.$) . In this case, the marginal benefit curve becomes$ the (dotted) horizontal line. Corollary 4 will later show (by proving the inequality $\left.B_{\rho \alpha}(\theta,)>0.\right)$ that $\rho^{*}(\theta)$ is in a positive relationship with $\alpha$, implying that the optimal level of investment in the case $\alpha=1$ is higher than in the case $\alpha \neq 1$, as also apparent from Figure 1.

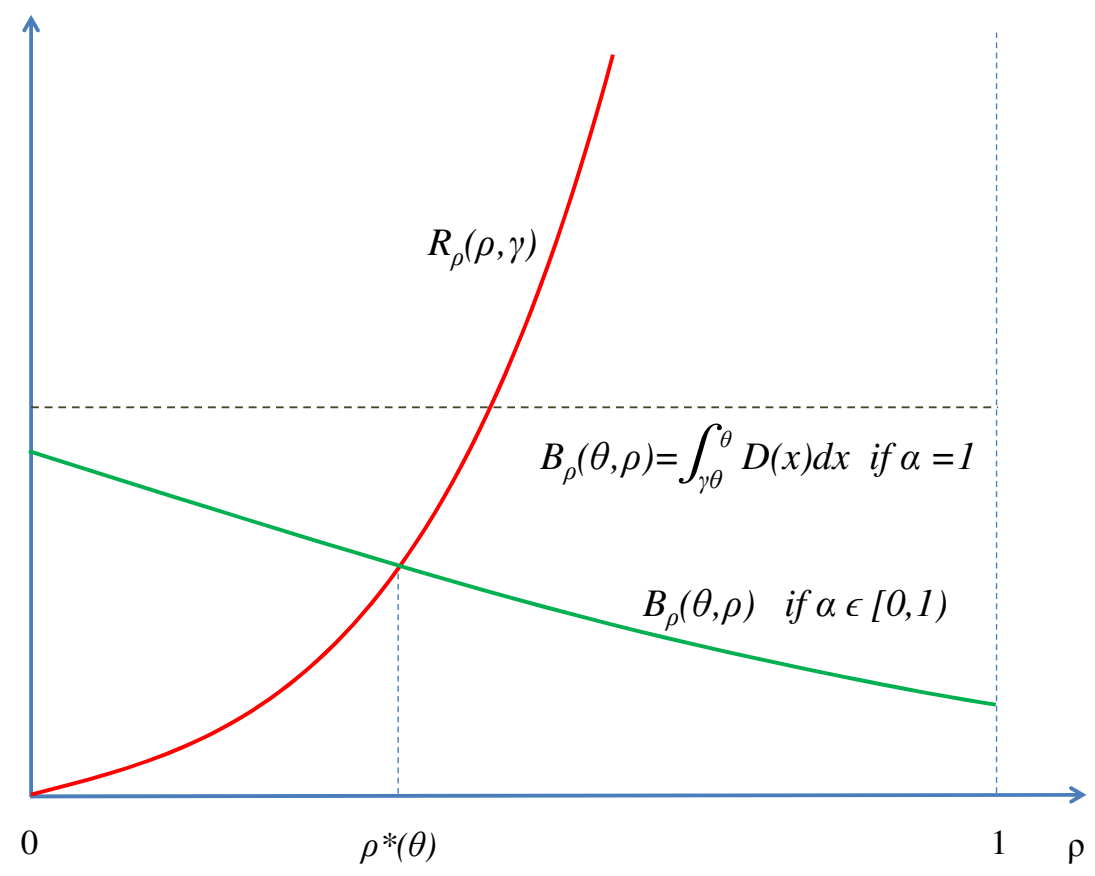

Figure 1. Observable Investment Decision of a Regulated Monopolist

Corollary 1 and Proposition 3 together allow us to identify the effect of adding observable investment into the B-M model on the optimal output schedule.

Proposition 4. Let Assumptions 2-8 and 10 hold and also Assumption 9-[ $\rho]$ hold for all $\rho \in[0,1)$. Then, the optimal output schedule in the integrated $B-M$ model with observable investment is always below the optimal output schedule in the B-M model without investment, i.e., $\bar{q}^{\rho^{*}(\theta)}(\theta)<\bar{q}(\theta)$ for all $\theta \in\left(0, \theta_{1}\right]$. 
The above result implies that the addition of observable investment decision to the BM model of regulation always reduces the ex-post output regardless whether the investment becomes successful or not. Below, we will examine how the optimal level of investment varies with the marginal cost. However, we need to first ensure that investment, when successful, will increase productive efficiency at the regulated output.

Assumption 11- $[\rho]$. Production costs are lower when investment is successful than when it is not; i.e., $C\left(\bar{q}^{\rho}(\gamma \theta), \gamma \theta\right)<C\left(\bar{q}^{\rho}(\theta), \theta\right)$ for all $\theta \in\left(0, \theta_{1}\right]$.

Corollary 2. Let Assumptions 2-8 and 10 hold and Assumptions 9-[ $\rho]$ and 11-[ $[\rho]$ hold for all $\rho \in[0,1)$. Then, the optimal level of observable investment, $\rho^{*}(\theta)$, is increasing in $\theta \in\left(0, \theta_{1}\right]$.

Interestingly, the above result implies that in regulatory environments where investment increases productive efficiency, a less efficient monopolist would always choose to invest more than a more efficient monopolist. Now we can explore the dependence of $\rho^{*}(\theta)$ on the parameter $\gamma$. For this, we need to estimate $B_{\rho \gamma}(\theta,$.$) ,$ the response of the marginal benefit schedule to a change in $\gamma$. Unfortunately, the impact of $\gamma$ on the partial derivative $\bar{q}_{\rho}^{\rho}($.$) appearing in the first and third integrals$ of (35) is indeterminate because of the ambiguous effect of $\gamma$ on the marginal informational cost function $F(. \mid \rho) / f(. \mid \rho)$ and its rate of change $\partial[F(. \mid \rho) / f(. \mid \rho)] / \partial \rho$. However, in situations where the welfare weight $\alpha$ is sufficiently close to 1 , the effects of these two terms on $\bar{q}^{\rho}($.$) and \partial \bar{q}^{\rho}(.) / \partial \rho$ become negligible. In such situations, the impact of $\gamma$ on $\rho^{*}(\theta)$ can be predicted, provided that the following assumption is also satisfied.

Assumption 12. $R_{\rho, \gamma}(\rho, \gamma)>0$ for all $\rho \in[0,1)$ and $\gamma \in(0,1)$ (marginal cost of investment is decreasing with the improvement level, i.e., increasing in $\gamma$ ).

Corollary 3. Let Assumptions 2-8 and 12 hold and Assumption 9-[$[\rho]$ hold for 
all $\rho \in[0,1)$. If $\alpha$ is sufficiently close to one, then for all values of $\theta \in(0, \theta]$, the optimal level of observable investment, $\rho^{*}(\theta)$, is increasing with the improvement level, i.e., decreasing in $\gamma \in(0,1)$.

The above result is intuitive once we observe (from the above discussion) that when $\alpha$ is sufficiently close to one, the effect of an increase in the improvement level of investment (or a decrease in $\gamma$ ) on the marginal benefit $B_{\rho}(\theta, \rho)$ can be approximated by the increase in the uncertain marginal benefit of investment, i.e., the increase in

the integral $\int_{\gamma \theta}^{\theta} \bar{q}^{\rho}(x) d x$ stemming from a decrease in $\gamma$, thanks to the negligibility of the first and third integrals in (35) when $\alpha$ is sufficiently high. Thus, we expect the curve $B^{\rho}(\theta, \rho)$ in Figure 1 to shift up when $\gamma$ decreases. On the other hand, the cost curve $R(\rho, \gamma)$ would shift down under Assumption 12, yielding an increase in $\rho^{*}(\theta)$.

In the next corollary, we show that when the social welfare is more equitable or the demand for the regulated product is higher, the monopolist will choose a higher level of investment.

Corollary 4. Let Assumptions 2-8 and 10 hold and Assumption 9-[$[\rho]$ hold for all $\rho \in[0,1)$. Then, for all values of $\theta \in\left(0, \theta_{1}\right]$, the optimal level of observable investment, $\rho^{*}(\theta)$, is increasing in both $\alpha \in[0,1]$ and $D_{0} \in(0, \infty)$.

It should be obvious from the optimal policy in (22)-(25) that the higher the welfare parameter $\alpha$ or the higher the maximal demand, $D_{0}$, the higher will be marginal informational rent at each cost level, and consequently the higher will be the marginal benefit of investment, implying a higher value for $\rho^{*}(\theta)$.

\subsection{Unobservable Investment}

Here, we will consider an environment where the regulator is not aware of the monopolist's investment technology $(\gamma, \rho)$. Additionally, the regulator cannot ex-post observe the investment made by the monopolist (i.e., the realized value of $\rho$ ). Naturally, in this environment the beliefs of the regulator about the marginal cost of production will always be equal to her prior beliefs, i.e., $f(. \mid 0)=f($.$) , and result-$ 
ingly the regulatory policy that she considers to be optimal will be $\langle\bar{r}, \bar{p}, \bar{q}, \bar{s}\rangle$, given by (13)-(16) calculated under the regulator's prior beliefs $f($.$) . Although the addition$ of the unobservable investment into the BM model of regulation will not change the regulatory policy schedules; it will change the realizations of these schedules at the reported cost of the monopolist if its investment becomes successful and decreases the marginal cost of the monopolist, say from $\theta$ to $\gamma \theta$. The likely change in the marginal cost of production will clearly affect the informational rent of the monopolist, too. Observing this, the monopolist can calculate its expected profit for each possible investment level and then determine its optimal investment.

To simplify the rest of our analysis, we will suppose that Assumption 9-[0] holds, implying that $\bar{r}()=$.1 . Now, let us fix $\theta \in\left(0, \theta_{1}\right]$ and $\gamma \in(0,1)$. When the level of investment is $\rho$, the profit expected by the monopolist can be written as

$$
\pi^{e}(\theta, \rho)=\rho \pi(\gamma \theta)+(1-\rho) \pi(\theta)-R(\rho, \gamma)
$$

or simply

$$
\pi^{e}(\theta, \rho)=B(\rho, \gamma)-R(\rho, \gamma)
$$

with

$$
B(\rho, \gamma)=\int_{\theta}^{\theta_{1}} \bar{q}(x) d x+\rho \int_{\gamma \theta}^{\theta} \bar{q}(x) d x
$$

denoting the expected benefit of the monopolist. Differentiating $\pi^{e}(\theta, \rho)$ with respect to $\rho$ yields

$$
\pi_{\rho}^{e}(\theta, \rho)=B_{\rho}(\rho, \gamma)-R_{\rho}(\rho, \gamma)=\int_{\gamma \theta}^{\theta} \bar{q}(x) d x-R_{\rho}(\rho, \gamma)
$$

Clearly, $\pi_{\rho}^{e}(\theta, 0)>0$ and $\lim _{\rho \uparrow 1} \pi_{\rho}^{e}(\theta, \rho)=-\infty$.

The monopolist will choose to make investment $(\rho>0)$ only if the resulting expected profit exceeds the profit under no investment $(\rho=0)$, i.e.,

$$
\pi^{e}(\theta, \rho) \geq \pi^{e}(\theta, 0)
$$

or equivalently

$$
\rho \int_{\gamma \theta}^{\theta} \bar{q}(x) d x-R(\rho, \gamma) \geq 0
$$


where we have used $R(0, \gamma)=0$ by Assumption 2. The above inequality requires that the expected additional informational rent is not below the average cost of investment, i.e.,

$$
\int_{\gamma \theta}^{\theta} \bar{q}(x) d x \geq \frac{R(\rho, \gamma)}{\rho} .
$$

Using this last condition, the monopolist's investment problem can be written as follows:

$$
\max _{\rho \in[0,1)} \pi^{e}(\theta, \rho) \quad \text { subject to }(44)
$$

We can now state our second characterization result.

Proposition 5. Let Assumptions 1-6 and 9-[0] hold. Then, for all $\theta \in\left(0, \theta_{1}\right]$, the optimal level of unobservable investment, $\rho^{*}(\theta)$, for the monopolist is unique, lies in $(0,1)$, and satisfies

$$
\int_{\gamma \theta}^{\theta} \bar{q}(x) d x=R_{\rho}\left(\rho^{*}(\theta), \gamma\right) .
$$

Figure 2 illustrates how the optimal investment level $\rho^{*}(\theta)$ balances the marginal benefit and marginal cost of investment. Here, the marginal benefit curve is always horizontal unlike in Figure 1. In fact, this horizontal curve always lies above the varying marginal benefit curve in Figure 1. This will enable us to compare the optimal levels of observable and unobservable investments, which we leave to Section 3.3.

The following result shows that our finding in Corollary 2, linking the optimal level of observable investment negatively to the productive efficiency, is actually independent of whether investment is observable or not.

Corollary 5. Let Assumptions 1-6, 9-[0], and 11-[0] hold. Then, the optimal level of unobservable investment, $\rho^{*}(\theta)$, is increasing in $\theta \in\left(0, \theta_{1}\right]$. 


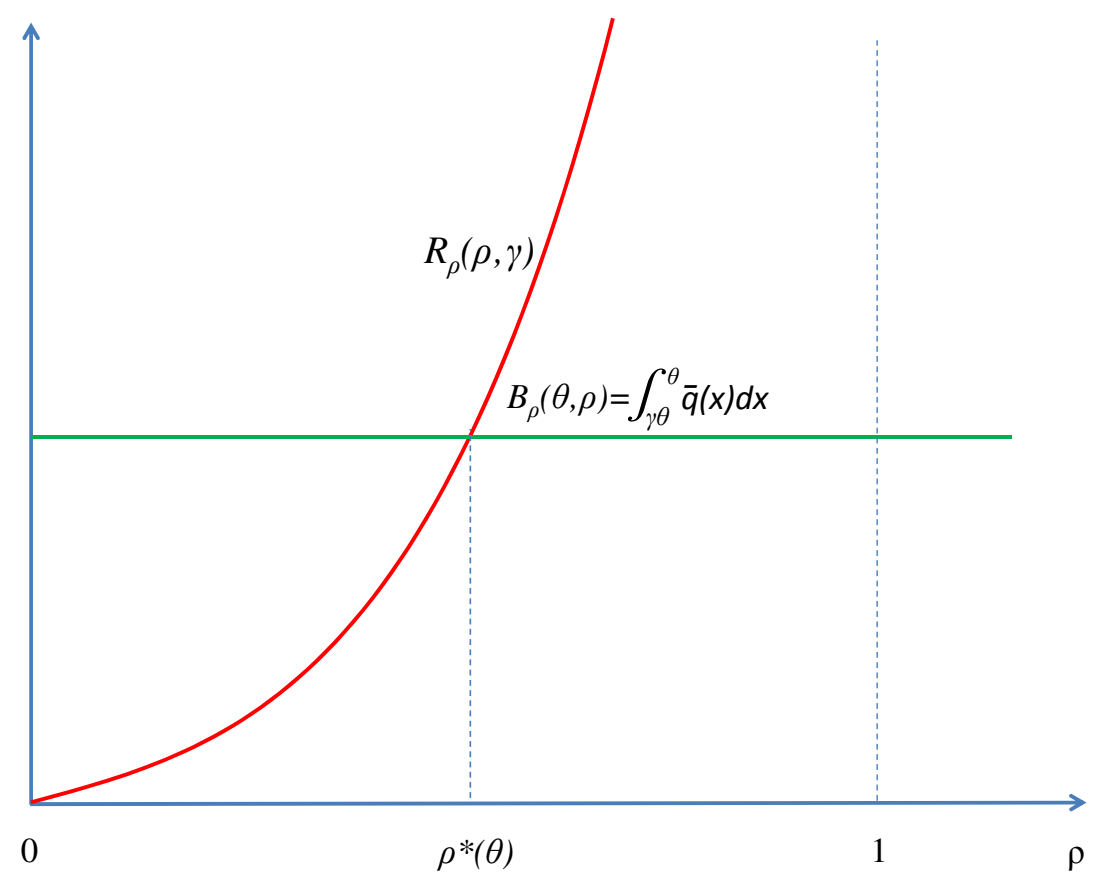

Figure 2. Unobservable Investment Decision of a Regulated Monopolist

Likewise, Corollary 6 will show together with Corollary 3 and 4 that the inability of the regulator to observe the investment of the monopolist has no effect on the direction of the relationship between the optimal level of investment and several parameters of our model, involving $\gamma, \alpha$, and $D_{0}$.

Corollary 6. Let Assumptions 1-6, 9-[0] and 12 hold. Then, for all $\theta \in\left(0, \theta_{1}\right]$, the optimal level of unobservable investment, $\rho^{*}(\theta)$, is increasing in $\alpha \in[0,1]$ and $D_{0} \in(0, \infty)$, while decreasing in $\gamma \in(0,1)$ (or increasing in the improvement level).

\subsection{Effect of Unobservability on the Investment Decision}

Now, we will explore how unobservability affects the investment decision of a regulated monopolist. Basically, we will compare the values of $\rho^{*}(\theta)$ calculated in Sections 3.1 and 3.2. This comparison will critically depend on whether the regulator weights the welfares of consumers and the monopolist equally or not. 
Proposition 6. Let Assumptions 2-8 and 10 hold and Assumption 9-[ $\rho]$ hold for all $\rho \in[0,1)$. Then for all $\theta \in\left(0, \theta_{1}\right]$, the optimal level of investment, $\rho^{*}(\theta)$, for the monopolist is (i) independent of the observability of investment if $\alpha=1$, (ii) lower when investment is observable than when it is unobservable if $\alpha \in[0,1)$.

Part (i) of the above result stems from the observation that with $\alpha=1$, we have $\bar{q}^{p}(\theta)=\bar{q}(\theta)=D(\theta)$. This implies that under Assumption 9-[0], the marginal benefits of investment are the same (as given by $B_{\rho}(\theta, \rho)=\int_{\gamma \theta}^{\theta} D(x) d x$ ) in Section 3.1 and Section 3.2. On the other hand, part (ii) of Proposition 6 follows from the fact that the marginal informational rent function (or the adjusted demand function) has lower values when investment is observable than when it is not, i.e., $\bar{q}^{\rho}()<.\bar{q}^{0}()=.\bar{q}($.$) for$ all $\rho \in(0,1)$, also implying lower marginal benefits of investment under observability. (The dotted horizontal line in Figure 1 corresponds to the marginal benefit curve for unobservable investment, which is always above the downward sloping marginal benefit curve for observable investment.)

We should also note that Proposition 6, along with Propositions 3 and 5, implies that for each $\theta \in\left(0, \theta_{1}\right]$, the optimal level of investment, $\rho^{*}(\theta)$, attains its maximal level when $\alpha=1$, i.e., whenever the outcome under the Baron and Myerson's (1982) regulatory policy essentially boils down to the outcome under Loeb and Magat's (1979) delegation scheme. The reason is that the monopolist in this particular case is entitled to the whole social surplus under the original demand curve (within the range of possible marginal costs). Thus, the (constant) marginal benefit of investment will be at its highest level, implying that for any level of the marginal cost the optimal investment will also be at its maximum.

Finally, our results in Section 3.1 and Section 3.2 also show that regardless whether investment is observable or not, the optimal level of investment, $\rho^{*}(\theta)$, is increasing in $\theta, \alpha$ and $D_{0}$ and decreasing in $\gamma$. 


\subsection{Effect of Regulation on the Investment Decision}

Our final goal is to estimate the impact of output regulation on the monopolist's investment decision. For this, we have to calculate first the optimal level of investment for the monopolist when the price and output of its product are not regulated.

Let us pick any $\theta \in\left(0, \theta_{1}\right]$. One can easily verify that when the unregulated monopoly does not make any investment, it would optimally choose the price and output of its product as $p^{m}(\theta)=\left(D_{0}+D_{1} \theta\right) / 2$ and $q^{m}(\theta)=\left(D_{0}-D_{1} \theta\right) / 2$, respectively. Resultingly, the monopolist's profit, $\pi^{m}(\theta)$, would become

$$
\pi^{m}(\theta)=p^{m}(\theta) q^{m}(\theta)-\theta q^{m}(\theta)-K=\frac{\left(D_{0}-D_{1} \theta\right)^{2}}{4 D_{1}}-K
$$

On the other hand, the profit the monopolist can expect under the investment technology $(\gamma, \rho)$ is equal to

$$
\pi^{m, e}(\theta, \rho)=\rho \pi^{m}(\gamma \theta)+(1-\rho) \pi^{m}(\theta)-R(\rho, \gamma)
$$

or simply

$$
\pi^{m, e}(\theta, \rho)=B(\theta, \rho)-R(\rho, \gamma)
$$

with

$$
B^{m}(\theta, \rho)=\rho \frac{\left(D_{0}-D_{1} \gamma \theta\right)^{2}}{4 D_{1}}+(1-\rho) \frac{\left(D_{0}-D_{1} \theta\right)^{2}}{4 D_{1}}-K
$$

denoting the expected benefit of the monopolist. The monopolist will choose to make investment $(\rho>0)$ if and only if

$$
\pi^{m, e}(\theta, \rho)-\pi^{m, e}(\theta, 0) \geq 0
$$

or equivalently

$$
\pi^{m}(\gamma \theta)-\pi^{m}(\theta) \geq \frac{R(\rho, \gamma)}{\rho}
$$

where we have used $R(0, \gamma)=0$ by Assumption 2. Thus, the monopolist's investment problem can be written as

$$
\max _{\rho \in[0,1)} \pi^{m, e}(\theta, \rho), \quad \text { subject to }(52)
$$


Noting that

$$
\begin{aligned}
\pi_{\rho}^{m, e}(\theta, \rho) & =B_{\rho}^{m}(\theta, \rho)-R_{\rho}(\rho, \gamma) \\
& =\frac{(1-\gamma) \theta}{2} D\left(\frac{(1+\gamma) \theta}{2}\right)-R_{\rho}(\rho, \gamma)
\end{aligned}
$$

and

$$
\pi_{\rho \rho}^{m, e}(\theta, \rho)=-R_{\rho \rho}(\rho, \gamma)
$$

we are ready to present our final characterization.

Proposition 7. Let Assumptions 2-6 hold. Then for all $\theta \in\left(0, \theta_{1}\right]$, the optimal level of investment, $\rho^{m}(\theta)$, for an unregulated monopolist is unique, lies in $(0,1)$, and satisfies

$$
\frac{(1-\gamma) \theta}{2} D\left(\frac{(1+\gamma) \theta}{2}\right)=R_{\rho}\left(\rho^{m}(\theta), \gamma\right)
$$

Using the characterizations provided by Propositions 5 and 7, we can compare the unobservable investment decision of a regulated monopolist to the investment decision of an unregulated monopolist, in situations where the regulator treats the welfares of consumer and the monopolist sufficiently equally.

Proposition 8. Let Assumptions 1-6 and 9-[0] hold. If $\alpha$ is sufficiently close to 1, then the optimal level of unobservable investment of a monopolist whose price and output are regulated is always higher than the optimal investment of an unregulated monopolist. That is, $\rho^{*}(\theta)$ satisfying (46) is higher than $\rho^{m}(\theta)$ satisfying (56).

Figure 3 illustrates the above result graphically. (Apparently, the intersection of the dotted horizontal line, depicting the curve for the marginal benefits of unobservable investment of a regulated monopolist, with the upward sloping marginal cost curve occurs at a higher value of $\rho$ than the optimal level of investment, $\rho^{m}(\theta)$, chosen by an unregulated monopolist.) The result in Proposition 8 is intuitive since in the 
extreme case where the regulator's objective attaches equal weights to the welfares of consumers and the monopolist, the outcome of the regulatory incentive-compatible policy used in the monopoly market coincides with the outcome of the delegation scheme of Loeb and Magat (1979), which entitles the monopolist to the whole social surplus at the sold output. This surplus always exceeds the unregulated monopoly profit, offering higher incentives to the monopolist for making investment when its production is regulated than when it is not.

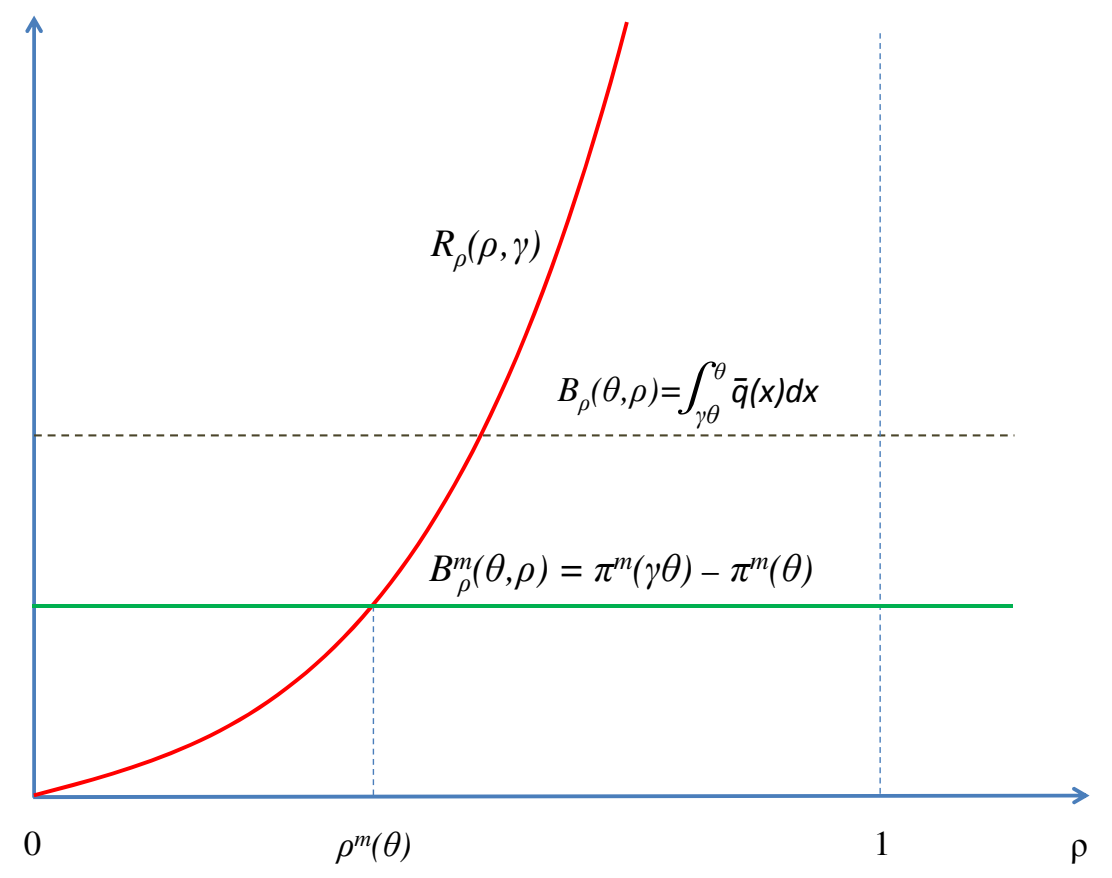

Figure 3. Investment Decision of an Unregulated Monopolist

On the other hand, in cases where the social welfare favors consumer welfare too much in relative to producer welfare (i.e., $\alpha$ is sufficiently small), it is not possible to compare the investment decision of the regulated monopolist to that of the unregulated monopolist even in the simpler situation where investment is unobservable. The reason is that under the regulatory policy (13)-(16), the adjusted demand schedule $\bar{q}($.$) affecting the informational rent of the monopolist nontrivially depends$ 
on the beliefs of the regulator through the inverse hazard rate function $F / f$, whose range may involve any positive real. However, it is also obvious that the lower the weight parameter $\alpha$ is, the higher will be the effect of the inverse hazard rate on the quantity schedule. In other words, the lower the parameter $\alpha$, the more suppressed the marginal benefit curve of the regulated monopolist, implying that the difference $\rho^{*}(\theta)-\rho^{m}(\theta)$ will also be lower.

\section{Conclusion}

This paper has studied whether the underinvestment effect of observability, earlier obtained by Tirole (1986) in a procurement model with investment, where both parties of the economic transaction, namely the buyer and the seller, have some asymmetric information, can also arise in a principal-agent model where 'only' the party that undertakes the investment, namely the agent, has some private information. The principal-agent model we have considered simply integrates investment with the monopoly regulation model of Baron and Myerson (1982). In this integrated framework, the regulator (principal) can contract on the price and output of the good produced by the monopolist (agent) but not on the investment the monopolist makes before production. We have considered both the case where investment is observable to the regulator and the case where it is not. For both cases, we have characterized the optimal level of investment chosen by the monopolist under some conditions ensuring the uniqueness and positiveness.

Irrespective of the observability issue, we have found that the optimal level of investment is higher when the monopolist is productively less efficient, provided that investment always increases productive efficiency. In addition, the improvement level of investment, the maximal size of demand and the relative weight of the monopolist welfare have, all, positive impacts on the optimal level of investment. However, the main result of our study is that the optimal level of investment is, in general, lower when investment is observable than when it is unobservable. Our result, along with the earlier result of Tirole (1986) in the same direction, reveals that the phenomenon of 'underinvestment due to observability' is independent of whether only the invest- 
ing firm or all of the parties affected by its investment decision have some private information about the post-investment economic relationship.

Why observability leads to underinvestment can be explained in our model by the well-known "ratchet effect" in the mechanism design literature, an effect similar to the 'information effect' offered by Tirole (1986) (which we have already addressed in Section 1). ${ }^{7}$ Basically, a contract that conditions (through the conditional beliefs of the regulator) on the level of investment is equivalent to ratcheting, whereby a monopolistic firm that demonstrates (through investment) that it is a lower cost supplier, will be required to supply at lower costs. Anticipating this, the firm chooses to invest less, to avoid the ratcheting up of its performance standards.

Our results also show that when the social welfare attaches a sufficiently high weight to the monopolist welfare, the unobservable investment of a regulated monopolist always exceeds the investment of an unregulated monopolist. This is because of the fact that when the deadweight loss of subsidy is negligible, the optimal subsidy schedule entitles the regulated monopolist to almost the whole social surplus, creating for it extremely high incentives for cost-reducing investment.

An important extension of our model would be the consideration of an environment where the regulator's observation about the likelihood of success - and or the improvement level - of investment is incomplete. Fruitfully, one can also consider environments where the regulator is authorized not only to control the price and output of the monopolistic product but also to control or influence its investment. We have implicitly assumed in our model that the regulator has limited ability (simply working through her beliefs) to influence the monopolist's investment because of some political or legal barriers or some conflicts of interests. Recall that in the case of fully observable investment, the regulator in our model is able to update her beliefs about the monopolist's costs (as $f\left(. \mid \rho^{*}\right)$ ) after observing the investment level, $\rho^{*}$, optimally chosen by the monopolist. However, although it may be subgame perfect for the regulator to resort to the B-M menu for the updated distribution, it is sub optimal to do so. In situations where the regulator has the full ability to control/determine the investment of the monopolist, she could set it at some socially

\footnotetext{
${ }^{7}$ See Weitzman (1980) and Freixas et al. (1985) for a discussion on the ratchet effect.
} 
efficient level, $\rho^{s}$, and could commit to the B-M menu corresponding to the belief distribution $f\left(. \mid \rho^{s}\right)$. In this way the regulator would use her commitment ability to achieve the desired investment. ${ }^{8}$ It may be interesting to check in that setup, the validity of a well-known proposition of Arrow (1959), claiming that an (unregulated) monopolist always has a lower incentive to innovate than a social planner and therefore its R\&D (investment) choice is socially suboptimal. In fact, in situations where the social welfare treats consumer and producer welfare equally $\alpha=1$, our results readily show that the regulated monopolist and the social planner would always have the same incentive to innovate, since irrespective of observability the regulatory output policy in this case would boil down to the policy consistent with marginal cost pricing, implying that the welfare (informational rent) of the monopolist becomes as high as the whole social surplus. On the other hand, it is also clear that in situations where $\alpha \neq 1$, the incentives of the regulated monopolist and the social planner would no longer be aligned because of the deadweight loss of subsidy, which is not internalized by the monopolist. Future study may explore whether in this case the unregulated investment activities of the regulated monopolist would be socially excessive or inadequate.

\section{References}

Arrow, K. J., 1959. "Economic Welfare and the Allocation of Resources for Invention," The Rand Corporation, rand Paper P-1856- RC. Republished as: "Economic Welfare and the Allocation of Resources to Invention," in R. R. Nelson (ed.), The Rate and Direction of Economic Activity, Princeton University Press, N.Y., 1962.

Baron, D., and Besanko, D., 1984. "Regulation and Information in a Continuing Relationship," Information Economics and Policy 1, 267-302.

Baron, D., and Myerson, R. B., 1982. "Regulating a Monopolist with Unknown Costs," Econometrica 50, 911-930.

\footnotetext{
${ }^{8}$ See Szalay (2005) for a principal-agent model where the principal uses his commitment ability to optimally influence the incentives of the agent.
} 
Freixas, X., Guesnerie, R., and Tirole, J., 1985. "Planning Under Incomplete Information and the Ratchet Effect," The Review of Economic Studies 52(2), 173-191.

Laffont, J.J., and Tirole, J., 1993. A Theory of Incentives in Procurement and Regulation, The MIT Press, Cambridge (Massachusetts).

Loeb, M., and Magat, W. A., 1979. "A Decentralized Method for Utility Regulation," Journal of Law and Economics 22, 399-404.

Sappington, D., 1982. "Optimal Regulation of Research and Development under Imperfect Information," The Bell Journal of Economics 13(2), 354-368.

Szalay, D., 2005. "The Economics of Clear Advice and Extreme Options," Review of Economic Studies 72(4), 1173-1198.

Tirole 1986. "Procurement and Renegotiation," Journal of Political Economy 94(2), 235-259.

Weitzman, M. L., 1980. "The "Ratchet Principle" and Performance Incentives," The Bell Journal of Economics 11(1), 302-308.

\section{Appendix}

Proof of Proposition 1. See pages 920-921 of Baron and Myerson (1982).

Proof of Lemma 1. Pick any $\rho \in[0,1)$. Assumption 7 ensures that $F(\theta \mid \rho) / f(\theta \mid \rho)$ is increasing in $\theta \in\left(0, \theta_{1}\right]$ if $\theta \neq \gamma \theta_{1}$. One can also check that

$$
\frac{F\left(\gamma \theta_{1} \mid \rho\right)}{f\left(\gamma \theta_{1} \mid \rho\right)}-\lim _{\theta \downarrow \gamma \theta_{1}} \frac{F(\theta \mid \rho)}{f(\theta \mid \rho)}=\frac{-f\left(\theta_{1}\right) F\left(\gamma \theta_{1}\right)-\frac{\rho}{(1-\rho)} f\left(\theta_{1}\right)}{f\left(\gamma \theta_{1}\right)\left[f\left(\theta_{1}\right)+\frac{\gamma(1-\rho)}{\rho} f\left(\gamma \theta_{1}\right)\right]}<0
$$

completing the proof.

Proof of Proposition 2. Directly obtained by mimicking the proof of Proposition 1 (thanks to Lemma 1). 
Proof of Lemma 2. Differentiating (20) with respect to $\rho$ yields

$$
\frac{\partial[F(\theta \mid \rho) / f(\theta \mid \rho)]}{\partial \rho}= \begin{cases}\frac{F(\theta / \gamma) f(\theta)-\frac{1}{\gamma} F(\theta) f(\theta / \gamma)}{[\rho f(\theta / \gamma)+(1-\rho) f(\theta)]^{2}} & \text { if } 0<\theta \leq \gamma \theta_{1}, \\ \frac{1}{(1-\rho)^{2} f(\theta)} & \text { if } \gamma \theta_{1}<\theta \leq \theta_{1} .\end{cases}
$$

The second line of the above derivative is always positive. Rewriting Assumption 8 for any $\theta \in\left(0, \gamma \theta_{1}\right]$ as $F(\theta) / f(\theta)<\gamma F(\theta / \gamma) / f(\theta / \gamma)$, we obtain that the first line of (58) is always positive, too. Thus $F(\theta \mid \rho) / f(\theta \mid \rho)$ is increasing in $\rho$, proving part (i). Also, by Assumption 7, $\partial[F(\theta \mid \rho) / f(\theta \mid \rho)] / \partial \rho$ is nondecreasing in $\rho$, proving convexity.

Proof of Corollary 1. Directly obtained from equations (22) and (23), given Lemma 2(i).

Proof of Remark 1. By Assumption 9-[0], Assumption 10 holds if (36) is satisfied. Pick any $\theta \in\left(0, \theta_{1}\right]$. We have $\bar{q}^{0}(\theta)=\bar{q}(\theta)$ and therefore, $\int_{\gamma \theta}^{\theta} \bar{q}^{0}(x) d x=\int_{\gamma \theta}^{\theta} \bar{q}(x) d x$, which is always positive, by equations (13) and (14). Now, let $\alpha=1$. For all $\rho \in[0,1), \bar{q}^{\rho}(\theta)=\bar{q}(\theta)=D(\theta)$, implying $\partial \bar{q}^{\rho}(\theta) / \partial \rho=0$. Thus, $B_{\rho}(\theta, 0)=\int_{\gamma \theta}^{\theta} \bar{q}(x) d x>0$. Since both $\bar{q}^{\rho}($.$) and \bar{q}_{\rho}^{\rho}($.$) are continuous in \alpha$, (36) holds for all $\alpha \in[0,1]$ which are sufficiently close to 1 .

Proof of Lemma 3. Pick any $\theta \in\left(0, \theta_{1}\right]$ and $\rho \in[0,1)$. Since Assumption 9- $[\rho]$ holds, $B_{\rho}(\theta, \rho)$ is given by $(35)$. Differentiating $B_{\rho}(\theta, \rho)$ with respect to $\rho$ yields

$$
B_{\rho \rho}(\theta, \rho)=\int_{\theta}^{\theta_{1}} \bar{q}_{\rho \rho}^{\rho}(x) d x+2 \int_{\gamma \theta}^{\theta} \bar{q}_{\rho}^{\rho}(x) d x+\rho \int_{\gamma \theta}^{\theta} \bar{q}_{\rho \rho}^{\rho}(x) d x .
$$

First let $\alpha \in[0,1)$. Thanks to Assumptions 7 and 8 , we have $\bar{q}_{\rho}^{\rho}(\theta)<0$ by Corollary 1 , and

$$
\bar{q}_{\rho \rho}^{\rho}(\theta)=D^{\prime}\left(\bar{p}^{\rho}(\theta)\right)(1-\alpha) \frac{\partial^{2}[F(\theta \mid \rho) / f(\theta \mid \rho)]}{\partial \rho^{2}} \leq 0,
$$


by Lemma 2-(ii). Therefore, $B_{\rho \rho}(\theta, \rho)<0$. Now let $\alpha=1$. Then, we have $q^{\rho}()=.\bar{q}()=.D($.$) , implying B_{\rho \rho}(\theta, \rho)=0$. Thus, for all $\alpha \in[0,1]$, we have $B_{\rho \rho}(\theta, \rho) \leq 0$. Moreover, we have $R_{\rho \rho}(\theta, \rho)>0$ by Assumption 6, implying $\pi_{\rho \rho}^{e}(\theta, \rho)<0$.

Proof of Proposition 3. Pick any $\theta \in\left(0, \theta_{1}\right]$. By Proposition 2 (thanks to Assumption 7 ), the optimal regulatory policy is given by (22)-(25). Note from equations (34) and (35) that under Assumption 9-[$]$, equation (37) is the first order condition for the problem in (33). Assumption 5 implies that $\rho^{*}(\theta)<1$. On the other hand, Assumptions 3-5 along with Assumption 10 and the continuity of $\pi^{e}(\theta, \rho)$ in $\rho$ imply that $\rho^{*}(\theta)>0$.

Now, pick any $\rho \in[0,1)$ and note that $\pi_{\rho \rho}^{e}(\theta, \rho)<0$ by Lemma 3 (thanks to Assumptions 6-8 and 9-[$[\rho])$. Thus, $\rho^{*}(\theta)$ satisfies the second-order condition and it is unique.

Finally, note that Assumptions 4 and 10 imply $\pi_{\rho}^{e}(\theta, 0)>0$, while the continuity of $B_{\rho}(\theta, \rho)$ and $R_{\rho}(\rho, \gamma)$ with respect to $\rho$ imply that $\pi_{\rho}^{e}(\theta, \rho)$ is continuous in $\rho$. Since we have already found that $\rho^{*}(\theta)$ is the unique maximizer of $\pi^{e}(\theta, \rho)$ among all $\rho \in[0,1)$, we must have $\pi^{e}\left(\theta, \rho^{*}(\theta)\right)-\pi^{e}(\theta, 0)>0$, ensuring the feasibility condition $(32)$.

Proof of Proposition 4. Due to the assumptions of the proposition, both Corollary 1 and Proposition 3 hold. Pick any $\theta \in\left(0, \theta_{1}\right]$. Corollary 1 implies $\bar{q}_{\rho}^{\rho}(\theta)<0$ for all $\rho \in[0,1)$. On the other hand, Proposition 3 ensures that $\rho^{*}(\theta)$ lies in $(0,1)$. Therefore, $\bar{q}^{\rho^{*}(\theta)}(\theta)<\bar{q}^{0}(\theta)=\bar{q}(\theta)$.

Proof of Corollary 2. Pick any $\theta \in\left(0, \theta_{1}\right]$. Since Assumptions 2-8 and 10 hold and Assumption 9- $[\rho]$ holds for all $\rho \in[0,1)$, Proposition 3 ensures that $\rho^{*}(\theta)$ is unique, lies in $(0,1)$, and satisfies $\pi_{\rho}^{e}\left(\theta, \rho^{*}(\theta)\right)=0$ as in (37). Now, pick any $\rho \in[0,1)$. By Assumption $9-[\rho], B_{\rho}(\theta, \rho)$ is given by (35). Differentiating (35) with respect to $\theta$ 
yields

$$
\begin{aligned}
B_{\rho \theta}(\theta, \rho) & =\bar{q}^{\rho}(\theta)-\gamma \bar{q}^{\rho}(\gamma \theta)+\rho\left[\bar{q}_{\rho}^{\rho}(\theta)-\bar{q}_{\rho}^{\rho}(\gamma \theta)\right]-\bar{q}_{\rho}^{\rho}(\theta) \\
& =\bar{q}^{\rho}(\theta)-\gamma \bar{q}^{\rho}(\gamma \theta)+(\rho-1) \bar{q}_{\rho}^{\rho}(\theta)-\rho \bar{q}_{\rho}^{\rho}(\gamma \theta) .
\end{aligned}
$$

First let $\alpha \in[0,1)$. By Corollary 1 (thanks to Assumptions 7 and 8), $\bar{q}_{\rho}^{\rho}(\theta)<0$. Now let $\alpha=1$. Then $\bar{q}_{\rho}^{\rho}(\theta)=0$, since $\bar{q}^{\rho}(\theta)=\bar{q}(\theta)=D(\theta)$. So, for all

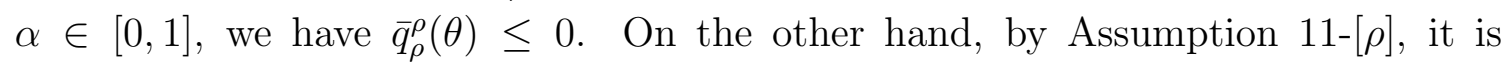
true that $C\left(\bar{q}^{\rho}(\theta), \theta\right)>C\left(\bar{q}^{\rho}(\gamma \theta), \gamma \theta\right)$, or equivalently $\bar{q}^{\rho}(\theta)>\gamma \bar{q}^{\rho}(\gamma \theta)$. Therefore, $B_{\rho \theta}(\theta, \rho)>0$, implying $\pi_{\rho \theta}^{e}(\theta, \rho)>0$ since $R_{\rho \theta}(\rho, \gamma)=0$. Additionally, for all $\rho \in[0,1], \pi_{\rho \rho}^{e, \rho, \gamma}(\theta)<0$ by Lemma 3 (thanks to Assumptions 6-8 and 9-[$[\rho]$ ). Since $\pi_{\rho}^{e}\left(\theta, \rho^{*}(\theta)\right)=0, \rho^{*}(\theta)$ must be increasing in $\theta \in\left(0, \theta_{1}\right]$.

Proof of Corollary 3. Pick any $\theta \in\left(0, \theta_{1}\right]$. Assumption 9-[0] implies Assumption 10. Since Assumptions 2-8 and 10 hold and Assumption 9- $[\rho]$ holds for all $\rho \in[0,1)$, Proposition 3 ensures that $\rho^{*}(\theta)$ is unique, lies in $(0,1)$, and satisfies $\pi_{\rho}^{e}\left(\theta, \rho^{*}(\theta)\right)=0$ as in (37). Now, pick any $\rho \in[0,1)$. First, let $\alpha=1$. From equations (22), (23), and (24) it follows that $\bar{q}^{\rho}(\theta)=\bar{q}(\theta)=D(\theta)$, hence $\bar{q}_{\rho}^{\rho}(\theta)=0$ for all $\theta \in\left(0, \theta_{1}\right]$. Since Assumption 9- $[\rho]$ holds, $\bar{r}^{\rho}(\theta)=1$ for all $\theta \in\left(0, \theta_{1}\right]$. Thus, equation (35) implies $B_{\rho}(\theta, \rho)=\int_{\gamma \theta}^{\theta} \bar{q}(x) d x$. It follows that $B_{\rho \gamma}(\theta, \rho)=-\theta \bar{q}(\gamma \theta)<0$; implying $\pi_{\rho \gamma}^{e}(\theta, \rho)=$ $-\theta \bar{q}(\gamma \theta)-R_{\rho \gamma}(\rho, \gamma)<0$ by Assumption 12. Moreover, for all $\rho \in[0,1), \pi_{\rho \rho}^{e}(\theta, \rho)<0$ by Lemma 3 (thanks to Assumptions 6-8 and 9-[$[\rho]$ ). Since $\pi_{\rho}^{e}\left(\theta, \rho^{*}(\theta)\right)=0, \rho^{*}(\theta)$ must be decreasing in $\gamma \in(0,1)$.

Finally, since $B_{\rho \gamma}(\theta, \rho)$ is continuous in $\alpha$ and the differences $\bar{q}^{\rho}()-.\bar{q}($.$) and$ $\bar{q}_{\rho}^{\rho}()-.\bar{q}_{\rho}()=.\bar{q}_{\rho}^{\rho}($.$) are negligible when 1-\alpha$ is sufficiently small, the above result obtained for $\alpha=1$ is also true for all $\alpha \in[0,1]$ which are sufficiently close to 1 .

Proof of Corollary 4. Pick any $\theta \in\left(0, \theta_{1}\right]$. Since Assumptions $2-8$ and 10 hold and Assumption 9- $[\rho]$ holds for all $\rho \in[0,1)$, Proposition 3 ensures that $\rho^{*}(\theta)$ is unique, lies in $(0,1)$, and satisfies $\pi_{\rho}^{e}\left(\theta, \rho^{*}(\theta)\right)=0$ as in (37). Now, pick any $\rho \in[0,1)$. Since Assumption 9- $[\rho]$ holds, $\bar{r}^{\rho}(\theta)=1$ for all $\theta \in\left(0, \theta_{1}\right]$. It follows from $(22)$ and $(23)$ 
that $\bar{q}^{\rho}(\theta)$ is increasing in both $\alpha \in[0,1]$ and $D_{0} \in(0, \infty)$. Moreover, we have

$$
\partial^{2} \bar{q}^{\rho}(\theta) / \partial \rho \partial \alpha=-D^{\prime}\left(\bar{p}^{\rho}(\theta)\right) \partial(F(\theta \mid \rho) / f(\theta \mid \rho)) / \partial \rho>0
$$

and

$$
\partial^{2} \bar{q}^{\rho}(\theta) / \partial \rho \partial D_{0}=0
$$

Then, it follows from (35) that $B_{\rho \alpha}(\theta, \rho)>0$ and $B_{\rho D_{0}}(\theta \rho)>0$, implying $\pi_{\rho \alpha}^{e}(\theta, \rho)>0$ and $\pi_{\rho D_{0}}^{e}(\theta, \rho)>0$. Moreover, for all $\rho \in[0,1), \pi_{\rho \rho}^{e, \rho, \gamma}(\theta)<0$ by Lemma 3 (thanks to Assumptions 6-8 and 9-[$[\rho]$ ). Since $\pi_{\rho}^{e}\left(\theta, \rho^{*}(\theta)\right)=0, \rho^{*}(\theta)$ must be increasing in both $\alpha \in[0,1]$ and $D_{0} \in(0, \infty)$.

Proof of Proposition 5. Pick any $\theta \in\left(0, \theta_{1}\right]$. By Assumption 1, the optimal regulatory policy is given by (13)-(16). Assumption 9-[0] implies that $\bar{r}()=$.1 . Then, (46) is the first order condition for the problem (45). The marginal benefit of investment $\int_{\gamma \theta}^{\theta} \bar{q}(x) d x$ is always positive by (13), and (14). Then, Assumptions 3 and 4 imply $\rho^{*}(\theta)>0$, whereas Assumption 5 implies $\rho^{*}(\theta)<1$. On the other hand, Assumptions 2 and 6 imply that $R_{\rho}\left(\rho^{*}(\theta), \gamma\right)>R\left(\rho^{*}(\theta), \gamma\right) / \rho^{*}(\theta)$; so (44) is satisfied at $\rho^{*}(\theta)$. Finally, the second order condition holds, since $\pi_{\rho \rho}^{e}(\theta, \rho)=-R_{\rho \rho}(\rho, \gamma)<0$ by Assumption 6. This also ensures that $\rho^{*}(\theta)$ is unique.

Proof of Corollary 5. Pick any $\theta \in\left(0, \theta_{1}\right]$. Since Assumptions 1-6 and 9-[0] hold, Proposition 5 ensures that $\rho^{*}(\theta)$ is unique, lies in $(0,1)$, and satisfies $\pi_{\rho}^{e}\left(\theta, \rho^{*}(\theta)\right)=0$ as in (46). By Assumption 1, the optimal regulatory policy is given by (13)-(16). Assumption 9-[0] implies that $\bar{r}()=$.1 . Now pick any $\rho \in\left(0, \theta_{1}\right]$. Differentiating (41) with respect to $\theta$ yields

$$
\pi_{\rho \theta}^{e}(\theta, \rho)=\bar{q}(\theta)-\gamma \bar{q}(\gamma \theta)
$$

which is always positive, since $\gamma<1$, Assumption 11-[0] holds, and $\bar{q}($.$) is de-$ creasing by (13) and (14), thanks to Assumption 1. Moreover, for all $\rho \in[0,1)$, $\pi_{\rho \rho}^{e}(\theta, \rho)=-R_{\rho \rho}(\rho, \gamma)<0$ by Assumption 6. Since $\pi_{\rho}^{e}\left(\theta, \rho^{*}(\theta)\right)=0, \rho^{*}(\theta)$ must be increasing in $\theta \in\left(0, \theta_{1}\right]$. 
Proof of Corollary 6. Pick any $\theta \in\left(0, \theta_{1}\right]$. Since Assumptions 1-6 and 9-[0] hold, Proposition 5 ensures that $\rho^{*}(\theta)$ is unique, lies in $(0,1)$, and satisfies $\pi_{\rho}^{e}\left(\theta, \rho^{*}(\theta)\right)=0$ as in (46). By Assumption 1, the optimal regulatory policy is given by (13)-(16). Assumption 9-[0] implies that $\bar{r}()=$.1 . Now pick any $\rho \in\left(0, \theta_{1}\right]$. Differentiating (41) with respect to $\gamma$ yields

$$
\pi_{\rho \gamma}^{e}(\theta, \rho)=-\theta \bar{q}(\gamma \theta)-R_{\rho \gamma}(\rho, \gamma)
$$

which is always negative, since Assumption 12 holds, $\theta>0, \gamma>0$, and $\bar{q}(\gamma \theta)>0$, by equations (13) and (14). On the other hand, for any $z \in\left\{\alpha, D_{0}\right\}$, differentiating (41) with respect to $z$ yields

$$
\pi_{\rho z}^{e}(\theta, \rho)=\int_{\gamma \theta}^{\theta} \bar{q}_{z}(x) d x
$$

which is always positive, since $\bar{q}($.$) is increasing in both \alpha$ and $D_{0}$ by equations (13) and (14). Moreover, for all $\rho \in[0,1)$, we have $\pi_{\rho \rho}^{e}(\theta, \rho)=-R_{\rho \rho}(\rho, \gamma)<0$ by Assumption 6. Since $\pi_{\rho}^{e}\left(\theta, \rho^{*}(\theta)\right)=0, \rho^{*}(\theta)$ must be decreasing in $\gamma \in(0,1)$ and increasing in both $\alpha \in[0,1]$ and $D_{0} \in(0, \infty)$.

Proof of Proposition 6. Let us first show part (i) holds. Let $\alpha=1$. Then, it follows from Propositions 1 and 2 that $\bar{q}^{\rho}(\theta)=\bar{q}(\theta)=D(\theta)$ for all $\theta \in\left(0, \theta_{1}\right]$. In that case, the profit $\pi^{e}(\theta, \rho)$ under both observable and unobservable investment is given by

$$
\pi^{e}(\theta, \rho)=\int_{\theta}^{\theta_{1}} D(x) d x+\rho \int_{\gamma \theta}^{\theta} D(x) d x-R(\rho, \gamma) .
$$

Thus, the optimal level of investment, will be the same irrespective of the observability of investment. Now, we will consider part (ii) of Proposition 6. Let $\alpha \in[0,1)$. Pick any $\theta \in\left(0, \theta_{1}\right], \gamma \in(0,1)$, and $\rho \in[0,1)$. Note from (41) that when investment is unobservable (Section 3.2), the marginal (expected) benefit becomes $B_{\rho}^{u n o b s}(\theta, \rho)=\int_{\gamma \theta}^{\theta} \bar{q}(x) d x$. On the other hand, when investment is observable (Section 3.1), the marginal benefit is equal to $B_{\rho}^{o b s}(\theta, \rho)=\int_{\theta}^{\theta_{1}} \bar{q}_{\rho}^{\rho}(x) d x+\int_{\gamma \theta}^{\theta} \bar{q}^{\rho}(x) d x+\rho \int_{\gamma \theta}^{\theta} \bar{q}_{\rho}^{\rho}(x) d x$, as was presented in equation (35). By Corollary 1, $\bar{q}_{\rho}^{\rho}()<$.0 . We also have $\bar{q}^{0}()=.\bar{q}($.$) , by equations$ 
(20), (22), and (23). Thus, $\bar{q}^{\rho}(.) \leq \bar{q}^{0}()=.\bar{q}($.$) . These observations imply that$ $B_{\rho}^{\text {obs }}(\theta, \rho)<\int_{\gamma \theta}^{\theta} \bar{q}(x) d x=B_{\rho}^{\text {unobs }}(\theta, \rho)$. Since $B_{\rho, \rho}^{\text {obs }}(\theta, \rho)<0$ and $B_{\rho, \rho}^{\text {unobs }}(\theta, \rho)=0$ for all $\rho \in[0,1)$, none of the two marginal benefit curves is ever upward sloping. On the other hand, irrespective of the observability of investment, we always have $R_{\rho, \rho}(\rho, \gamma)>0$ for all $\rho \in[0,1)$, implying that the marginal cost curve is everywhere upward sloping. Since $\rho^{*}(\theta)$ is found at the intersection of the marginal benefit and the marginal cost curves, and since the curve $B_{\rho}^{\text {obs }}(\theta,$.$) everywhere lies below$ $B_{\rho}^{\text {unobs }}(\theta,),. \rho^{*}(\theta)$ must be lower when investment is observable than when it is unobservable.

Proof of Proposition 7. Pick any $\theta \in\left(0, \theta_{1}\right]$. Note that equation (56) is the first order necessary condition $\pi_{\rho}^{m, e}\left(\theta, \rho^{m}(\theta)\right)=0$ for an interior solution to the problem in (53). Assumptions 3 and 4 imply $\rho^{*}(\theta)>0$, since the left hand side of (56) is always positive. On the other hand, Assumption 5 implies that $\rho^{*}(\theta)<1$. Finally, Assumptions 2 and 6 together imply that $R_{\rho}\left(\rho^{m}(\theta), \gamma\right)>R\left(\rho^{m}(\theta), \gamma\right) / \rho^{m}(\theta)$; so (52) is satisfied at $\rho^{m}(\theta)$. Finally, given equation (55) and Assumption 6, it is obvious that $\rho^{m}(\theta)$ satisfies the second-order condition and it is unique.

Proof of Proposition 8. Pick any $\theta \in\left(0, \theta_{1}\right]$. Since Assumptions 1-6 and 9[0] hold, Proposition 5 ensures that the optimal investment, $\rho^{*}(\theta)$, of the regulated monopolist is unique, lies in $(0,1)$, and satisfies equation (46). Also, since investment is unobservable, the optimal regulatory policy is given by (13)-(16), by Assumption 1. First let $\alpha=1$. Then, $\bar{q}(x)=D(x)$ for all $x \in\left(0, \theta_{1}\right]$. It follows from (41) that for all $\rho \in[0,1)$

$$
B_{\rho}(\theta, \rho)=\int_{\gamma \theta}^{\theta} \bar{q}(x) d x=\int_{\gamma \theta}^{\theta}\left(D_{0}-D_{1} x\right) d x=(1-\gamma) \theta D\left(\frac{(1+\gamma) \theta}{2}\right) .
$$

So, $B_{\rho}(\theta, \rho)=2 B_{\rho}^{m}(\theta, \rho)$ for all $\rho \in[0,1)$. Using optimality conditions $B_{\rho}\left(\theta, \rho^{*}(\theta)\right)=$ $R_{\rho}\left(\rho^{*}(\theta), \gamma\right)$ and $B_{\rho}^{m}\left(\theta, \rho^{m}(\theta)\right)=R_{\rho}\left(\rho^{m}(\theta), \gamma\right)$, along with the fact $B_{\rho, \rho}(\theta, \rho)=$ $B_{\rho, \rho}^{m}(\theta, \rho)=0$ and Assumption 6, we can conclude that $\rho^{*}(\theta)>\rho^{m}(\theta)$. Now consider $\alpha \neq 1$. Since $\bar{q}($.$) is continuous in \alpha, B_{\rho}(\theta, \rho)$ will be continuous, too. Thus, the proof for $\alpha=1$ will also be valid for all $\alpha \in[0,1]$ sufficiently close to 1 . 